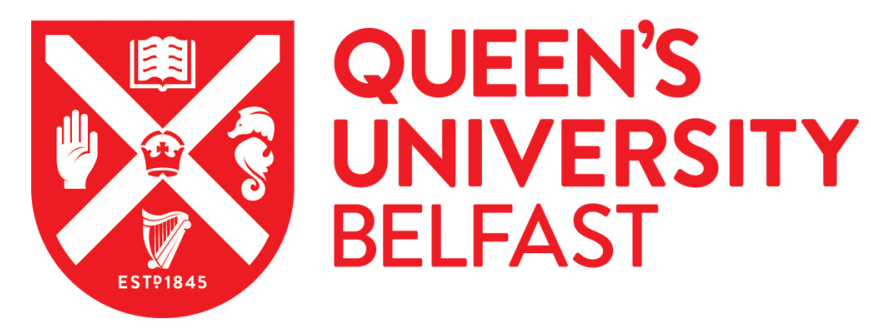

\title{
Local conductance: A means to extract polarization and depolarizing fields near domain walls in ferroelectrics
}

Douglas, A. M., Kumar, A., Whatmore, R. W., \& Gregg, J. M. (2015). Local conductance: A means to extract polarization and depolarizing fields near domain walls in ferroelectrics. Applied Physics Letters, 107, [172905]. https://doi.org/10.1063/1.4934833

Published in:

Applied Physics Letters

Document Version:

Publisher's PDF, also known as Version of record

Queen's University Belfast - Research Portal:

Link to publication record in Queen's University Belfast Research Portal

\footnotetext{
General rights
Copyright for the publications made accessible via the Queen's University Belfast Research Portal is retained by the author(s) and / or other copyright owners and it is a condition of accessing these publications that users recognise and abide by the legal requirements associated with these rights.
}

Take down policy

The Research Portal is Queen's institutional repository that provides access to Queen's research output. Every effort has been made to ensure that content in the Research Portal does not infringe any person's rights, or applicable UK laws. If you discover content in the Research Portal that you believe breaches copyright or violates any law, please contact openaccess@qub.ac.uk. 


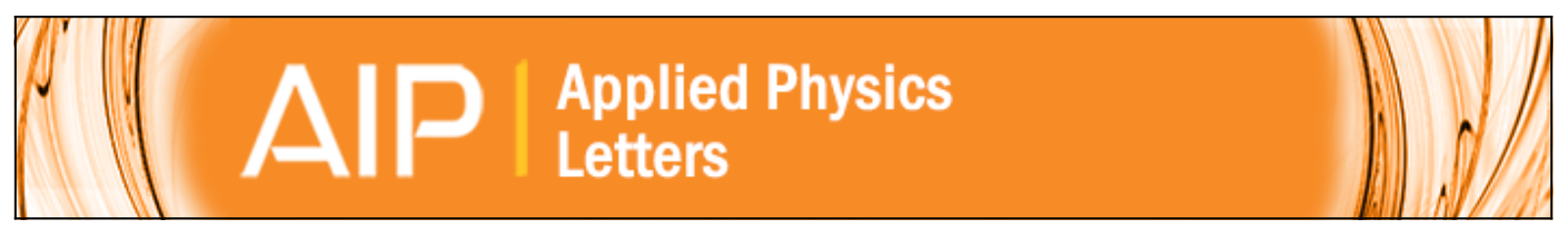

\section{Local conductance: A means to extract polarization and depolarizing fields near domain walls in ferroelectrics}

A. M. Douglas, A. Kumar, R. W. Whatmore, and J. M. Gregg

Citation: Applied Physics Letters 107, 172905 (2015); doi: 10.1063/1.4934833

View online: http://dx.doi.org/10.1063/1.4934833

View Table of Contents: http://scitation.aip.org/content/aip/journal/apl/107/17?ver=pdfcov

Published by the AIP Publishing

\section{Articles you may be interested in}

Domain wall stability in ferroelectrics with space charges

J. Appl. Phys. 115, 084110 (2014); 10.1063/1.4866359

Polarization reversal and jump-like domain wall motion in stoichiometric LiTaO3 produced by vapor transport equilibration

J. Appl. Phys. 111, 014101 (2012); 10.1063/1.3673601

Surface effect on domain wall width in ferroelectrics

J. Appl. Phys. 106, 084102 (2009); 10.1063/1.3236644

Charge and polarization distributions at the $90^{\circ}$ domain wall in barium titanate ferroelectric

Appl. Phys. Lett. 89, 182903 (2006); 10.1063/1.2374676

Decay of ferroelectric domains formed in the field of an atomic force microscope

J. Appl. Phys. 97, 084111 (2005); 10.1063/1.1862761

\section{AIP $\left.\right|_{\text {APL Photonics }}$}

APL Photonics is pleased to announce Benjamin Eggleton as its Editor-in-Chief

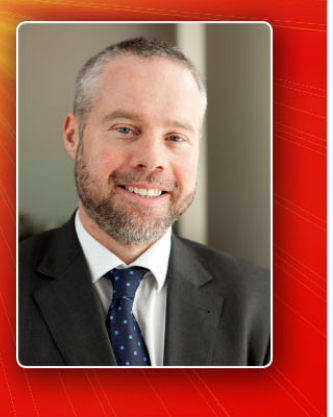




\title{
Local conductance: A means to extract polarization and depolarizing fields near domain walls in ferroelectrics
}

\author{
A. M. Douglas, ${ }^{1}$ A. Kumar, ${ }^{1}$ R. W. Whatmore, ${ }^{2}$ and J. M. Gregg ${ }^{1}$ \\ ${ }^{1}$ Centre for Nanostructured Media, School of Mathematics and Physics, Queen's University Belfast, \\ University Road, Belfast BT7 INN, United Kingdom \\ ${ }^{2}$ Department of Materials, Imperial College London, Exhibition Road, London SW7 2AZ, United Kingdom
}

(Received 14 August 2015; accepted 17 October 2015; published online 27 October 2015)

\begin{abstract}
Conducting atomic force microscopy images of bulk semiconducting $\mathrm{BaTiO}_{3}$ surfaces show clear stripe domain contrast. High local conductance correlates with strong out-of-plane polarization (mapped independently using piezoresponse force microscopy), and current-voltage characteristics are consistent with dipole-induced alterations in Schottky barriers at the metallic tip-ferroelectric interface. Indeed, analyzing current-voltage data in terms of established Schottky barrier models allows relative variations in the surface polarization, and hence the local domain structure, to be determined. Fitting also reveals the signature of surface-related depolarizing fields concentrated near domain walls. Domain information obtained from mapping local conductance appears to be more surface-sensitive than that from piezoresponse force microscopy. In the right materials systems, local current mapping could therefore represent a useful complementary technique for evaluating polarization and local electric fields with nanoscale resolution. (C) 2015 AIP Publishing LLC.

[http://dx.doi.org/10.1063/1.4934833]
\end{abstract}

Since their invention, differences in the physical principles underlying image formation in Scanning Tunneling and Atomic Force Microscopies (STM and AFM) ${ }^{1,2}$ have resulted in preconceived notions about the material characteristics that each technique can probe: transport behavior and electronic structure have been the traditional preserve of STM, while AFM has been almost exclusively associated with monitoring variations in tip-sample interaction forces. Perhaps this is why the simple realization that conducting AFM (cAFM) tips could also be used to monitor spatial variations in sample conductivity has been remarkably slow to emerge. Yet, cAFM has been found to be particularly useful in certain circumstances: for example, in identifying minority conducting phases embedded in an insulating matrix ${ }^{3}$ as demonstrated by the recent discovery of conducting domain walls in ferroelectrics and multiferroics. ${ }^{4-9}$ Here, STM is completely unsuitable, as the vast majority of the sample is insulating.

The magnitudes of local currents measured using cAFM are affected by both the transport characteristics of the material under investigation and the nature of the tip-sample contact. Schottky barriers are often expected; indeed, current variations can sometimes have little to do with differences in inherent sample conductivity, being dominated instead by localized alterations in barrier potentials. ${ }^{10-15} \mathrm{~A}$ striking illustration is in giant tunneling electroresistance (TER), where changes in the interfacial tunneling barrier height, caused by variations in electrical polarization in different ferroelectric domains, can result in an alteration of the measured resistance of up to $75000 \% .{ }^{11}$ Non-volatile binary memory and memristors based on this remarkable effect have been suggested. ${ }^{14,15}$ While the absolute contrast, in measured currents as a function of ferroelectric polarization orientation, is maximized in ultrathin films, it is also present when performing cAFM on bulk samples and becomes obvious when the conductivity of the bulk material is sufficiently high. This has been demonstrated in $\mathrm{HoMnO}_{3}{ }^{15}$ and will be further illustrated here.

In this paper, we show that $\mathrm{AFM}$ contrast on polished surfaces of bulk semiconducting $\mathrm{BaTiO}_{3}$ is strongly related to the out-of-plane polarization, inferred from piezoresponse force microscopy (PFM). Local current-voltage transport characteristics are consistent with the existence of Schottky barriers and established models can be used to extract parameters proportional to the out-of-plane component of polarization in different domains. There is even evidence that the cAFM signal contains information concerning the depolarizing fields around the surface traces of domain walls. As might be expected, the polarization detected through cAFM is strongly surface sensitive (more so than PFM). This illustrates the potential for CAFM in the nanoscale mapping of polarization, as well as the resistive switching potential of ferroelectric semiconductors, even in bulk.

Throughout this study, doped barium titanate ceramic samples, used in commercial Positive Temperature Coefficient of Resistance (PTCR) devices (Amphenol YS5139 and YS6942), were examined. Quantitative energy dispersive x-ray characterization was performed and indicated the following compositions: $\left(\mathrm{Ba}_{0.85 \pm 0.01} \mathrm{Ca}_{0.150 \pm 0.002}\right) \mathrm{Ti}_{0.93 \pm 0.1} \mathrm{O}_{3}$ (YS5139) and $\left(\mathrm{Ba}_{0.68 \pm 0.02} \mathrm{Ca}_{0.115 \pm 0.002} \mathrm{~Pb}_{0.161 \pm 0.003}\right) \mathrm{Ti}_{0.92 \pm 0.02} \mathrm{O}_{3}$ (YS6942). The ceramics were found to be chemically homogeneous, save for slight stoichiometric variations across a few unit cells adjacent to grain boundaries. The ceramics have a relatively low bulk resistivity at room temperature (typically $10^{1}-10^{2} \Omega \mathrm{cm}$ ), but this increases dramatically, by up to 4 orders of magnitude, on heating through the ferroelectric Curie temperature (see Figure 1(a) for the bulk resistance-temperature variation in the two ceramics studied here). This phenomenon is thought to arise from the way in which polarization can locally reduce the resistivity of grain 
(a)

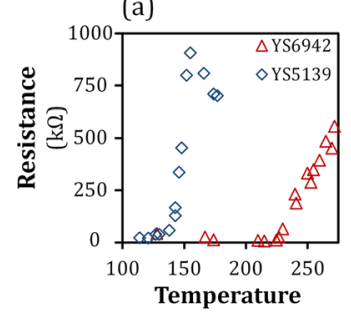

(e)

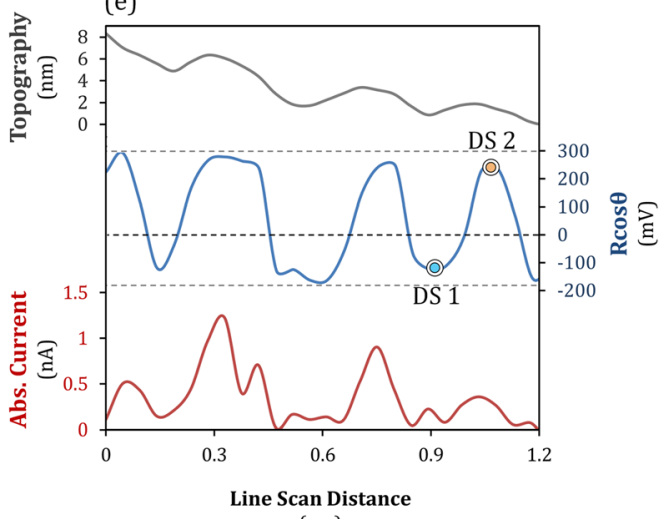

$(\mu \mathrm{m})$ (b)

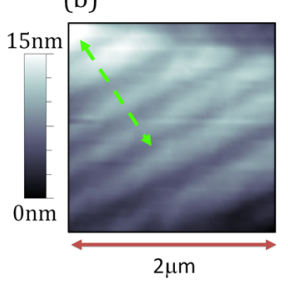

(c)

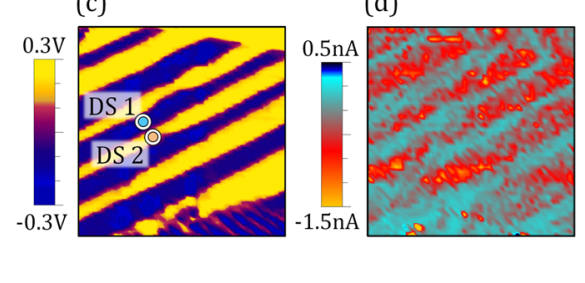

(d)

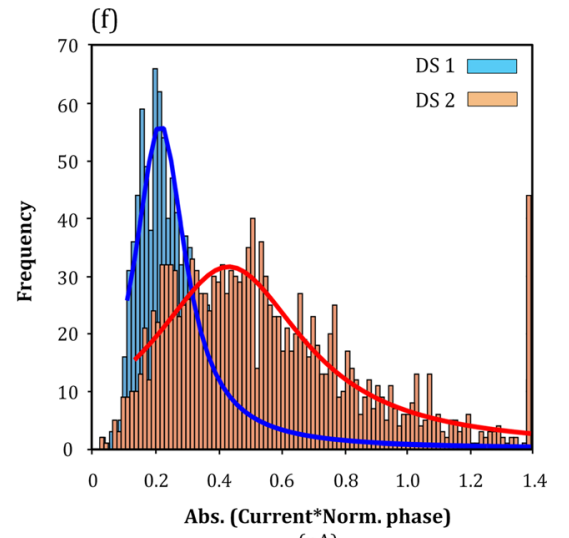

(nA)

FIG. 1. Macroscopic measurement of the resistance variation with temperature in the semiconducting $\mathrm{BaTiO}_{3}$ ceramics examined in this study (a). Imaging of a chemically-mechanically polished ceramic surface shows slight topographic variation (b) which correlates strongly with both ferroelectric domain information from vertical piezoresponse force microscopy (PFM; represented here as Rcos $\theta$, or amplitude multiplied by the cosine of the phase (c)) and local currents measured using conducting atomic force microscopy (cAFM (d)). The line sections (e), taken from each SPM image in the direction shown by the green dashed line in (b), reiterate these correlations. A quantitative analysis, in which the PFM phase information at each pixel is renormalized to be either +1 (for positive phase) or -1 (for negative phase) and then multiplied by the value of current measured at that pixel, shows explicitly that high current in the stripe domains correlates with out-of-plane polarization (f). DS 1 and DS 2 are the two domain states, with opposite senses of polarization perpendicular to the polished surface (DS 1: into-the plane; DS 2: out-of-the-plane).

boundaries. The samples of ceramic were cut and chemically-mechanically polished using an alkaline alumina suspension, and their local characteristics were investigated using a number of different scanning probe microscopy (SPM) techniques. All SPM was performed on a Dimension 3100 $\mathrm{AFM}$ at room temperature, using $\mathrm{Pt} / \mathrm{Ir}$ coated silicon tips (Nanosensors). Atomic Force Microscopy (AFM) was performed in tapping mode, Piezoresponse Force Microscopy (PFM) was performed at $20 \mathrm{kHz}$ in contact, and an extended TUNA module was used for cAFM using dc voltage, again in contact mode. AFM showed the resulting surfaces to be smooth (Figure 1(b)), save for slight topographic variations associated with preferential etching of different domain variants; the link between domain orientation and topography was made obvious when the vertical PFM maps (displayed as the amplitude multiplied by the cosine of the phase, $\mathrm{R} \cos \theta$, in Figure 1(c)) and AFM images were compared. As can also be seen in Figure 1(d), cAFM often produced current contrast that reflected both the domain structure and surface topography images. This was not due to topographic cross-talk: the absolute differences in height between the adjacent domains was of the order of nanometers and, within each domain, the RMS surface roughness was usually subnanometre.

The correlation between AFM, PFM, and cAFM data has been further illustrated, in qualitative terms, in Figure 1(e). Here, the domains with slightly elevated surface topography can be seen to correlate with out-of-plane (rather than into the plane) vertical polarization components and enhanced local currents. Qualitatively, the link between polarization and current can be understood as a result of the proximity of the positive end of the electrical dipole to the tip-ferroelectric interface: it should locally lower electron energy states, decreasing the expected Schottky barrier height and increasing observed conduction. ${ }^{15}$ For a more robust quantitative analysis of the correlation between the polar orientation and the local current, the phase information obtained from the vertical PFM was normalized such that in-phase piezoresponse (indicating that the vertical component of polarization is out-of-the-plane) was assigned a value of +1 and out-ofphase piezoresponse (indicating that the vertical component of polarization is into-the-plane) a value of -1 . At every pixel, in the images in Figures 1(c) and 1(d), the measured local current was multiplied by this normalized value of the PFM phase $( \pm 1)$ and the resulting positive and negative current value distributions plotted in Figure 1(f) (and fitted to Lorentzian functions). While the two distributions do have overlap, it is clear that, on the whole, the currents expected in pixels with polarization oriented out-of-the-plane are larger than those oriented into-the plane.

To investigate further, we used the CAFM to take a series of current-voltage $(I-V)$ measurements across the width of another set of two stripe domains. The resulting $I-V$ curves as a function of position are shown in Figure 2. To see the extent to which such $I-V$ behavior can provide information on the local out-of-plane polarization, we employed a Schottky barrier analysis.

Quantitatively, the effects of dipole orientation on barrier height, and consequently on current densities as a function of applied voltage, have already been considered by 
(a)

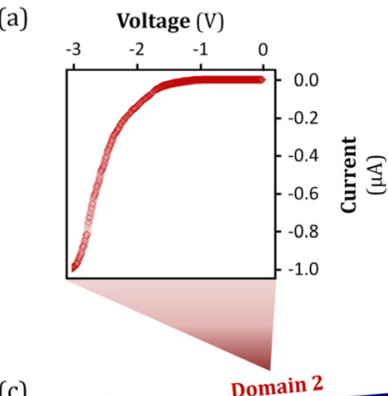

(b)

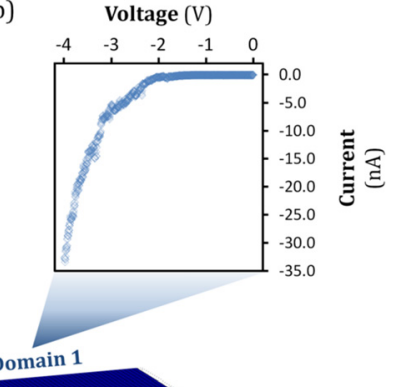

(c)

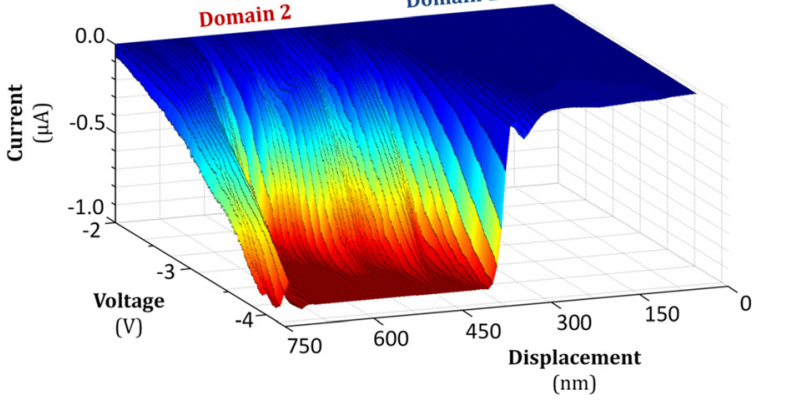

FIG. 2. Current was measured as a function of voltage $(I-V)$ using cAFM at a series of 50 points with a separation of $15 \mathrm{~nm}$ across a pair of stripe domains. Representative I-V curves for each domain are shown in (a) and (b) and all 50 curves have been combined to generate a surface plot (c) of current as a function of both spatial position and applied voltage.

Pintilie and Alexe ${ }^{16,17}$ among others. Under thermionic emission conditions, probably relevant at room temperature, the current density $(J)$ across the Schottky barrier is traditionally described by the following equation:

$$
J=A^{*} T^{2} e^{-\frac{q}{k T} \phi_{b}} e^{\frac{q}{T T} \Delta \phi_{b}},
$$

where $A^{*}$ is the Richardson constant, $T$ is the temperature, $q$ is the electron charge, $k$ is the Boltzmann constant, $\phi_{b}$ is the potential barrier in the absence of applied voltage $(V)$ or ferroelectric polarization components perpendicular to the tip-surface interface $(P)$, and $\Delta \phi_{b}$ is the alteration in the potential barrier due to $V$ and $P$. According to literature, ${ }^{16,17}$ $\Delta \phi_{b}$ can be given as

$$
\Delta \phi_{b}=\sqrt{\frac{q}{4 \pi \varepsilon_{0} \varepsilon_{o p}}}\left\{\left[\frac{2 q N_{e f f}}{\varepsilon_{0} \varepsilon_{s}}\left(V+V_{b i}^{\prime}\right)\right]^{1 / 2}+\frac{P}{\varepsilon_{0} \varepsilon_{s}}\right\}^{1 / 2},
$$

where $\varepsilon_{0}$ is the permittivity of vacuum, $\varepsilon_{\mathrm{op}}$ and $\varepsilon_{\mathrm{s}}$ are the relative permittivities of the ferroelectric at high (optical) and low (static) frequencies, respectively, $N_{\text {eff }}$ is the effective density of charge in the depletion region, and $V_{b i}^{\prime}$ is the apparent built-in potential, which is given by

$$
V_{b i}^{\prime}=V_{b i}+\frac{P}{\varepsilon_{0} \varepsilon_{s}} \delta
$$

where $\delta$ is the distance between the polarization surface charge and the physical interface with the electrode and is typically of the order of the unit cell length.

At a constant temperature, the combined terms $A^{*} T^{2} e^{-\frac{q}{k T} \phi_{b}}$ can be given as a constant $(K)$ (although in some circumstances the Richardson constant is known to vary), ${ }^{18}$ and Equations (1) and (2) can be combined to generate the following expression in which $\left[\ln \left(\frac{J}{K}\right)\right]^{2}$ is a linear function of $\left(V+V_{b i}^{\prime}\right)^{\frac{1}{2}}$ :

$$
\begin{aligned}
{\left[\ln \left(\frac{J}{K}\right)\right]^{2}=} & \frac{\sqrt{2 q^{7} N_{e f f}}}{k^{2} T^{2} 4 \pi \varepsilon_{0} \varepsilon_{o p} \sqrt{\varepsilon_{0} \varepsilon_{s}}}\left(V+V_{b i}^{\prime}\right)^{\frac{1}{2}} \\
& +\frac{q^{3}}{k^{2} T^{2} 4 \pi \varepsilon_{0}^{2} \varepsilon_{o p} \varepsilon_{s}} P .
\end{aligned}
$$

Here, the intercept is directly indicative of the out-of-plane polarization.

To test the validity of this linear expression and how it could be used to probe local out-of-plane polarization from cAFM mapping, we applied it to $I-V$ data from Figure 2. As a proxy for Equation $(4),(\ln I)^{2}$ was plotted against $-|V|^{1 / 2}$. In general, this should not be a linear function, unless $V \gg V^{\prime}{ }_{b i}$. The difficulty is that $V_{b i}^{\prime}$ is not known, and, in any case, it varies as a function of the local out-of-plane polarization. We have, therefore, taken the pragmatic approach of identifying the linear portions of the plots of $(\ln I)^{2}$ against $-|V|^{1 / 2}$ and, by virtue of their linearity, concluded that within these specific regions the $V \gg V_{b i}^{\prime}$ condition is satisfied. Approximately linear regions in $(\ln l)^{2}$ against $-|V|^{1 / 2}$ for each domain are plotted in Figures 3(a) and 3(b).

Across the $I-V$ measurements taken, the gradients of these plots varied, but they did show distinctly different distributions for each domain (Figure 3(c)). Taking the mean value of the gradient for each domain as representative of those associated with the polarizations within each domain, extrapolated intercept values for $(\ln I)^{2}$ at $V=0$ were extracted for all of the $I-V$ measurements taken. These intercept values, which should be indicative of local polarization (Equation 4), are plotted in Figure 3(d) as a function of position. Several points can be noted: first, the domain determined to have larger positive (out-of-the plane) polarization, from analysis of currents only, is also the more conducting domain, consistent with the correlations between cAFM and (a)

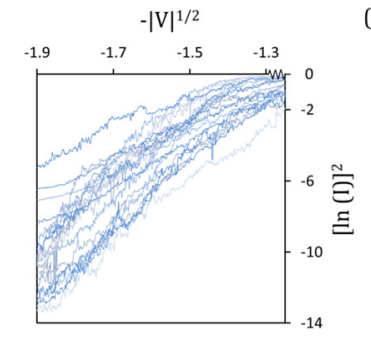

(c)

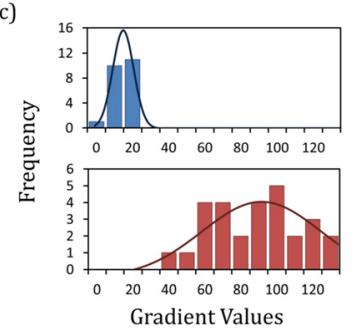

$\square$ Domain 1 (b)

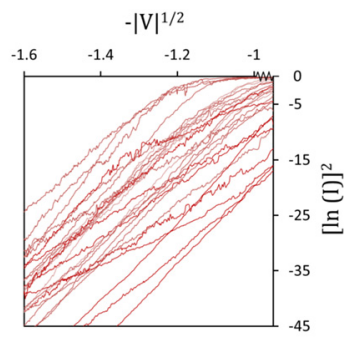

(d)

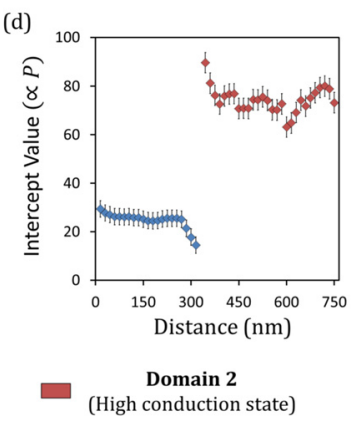

FIG. 3. Values of $(\ln l)^{2}$ were plotted as a function of $-|V|^{1 / 2}$ for each domain mapped in Figure 2, and the linear regions are presented in (a) and (b). Gradients of these linear regions were clearly bimodal (c) reflecting the very different conduction behavior in the two domains. With reference to standard models for thermionic Schottky emission, variations in the extrapolated intercept values (at $V=0$ ) from these linear plots should directly reflect spatial variations in polarization $(\mathrm{d})$. 
(a)

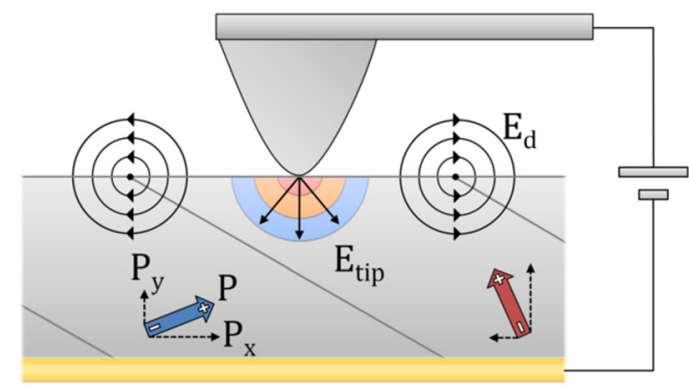

(b)

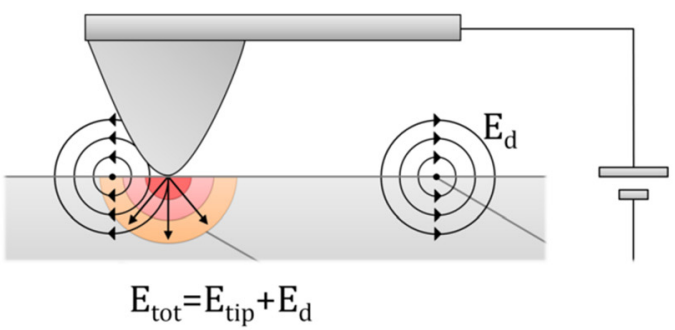

(c)

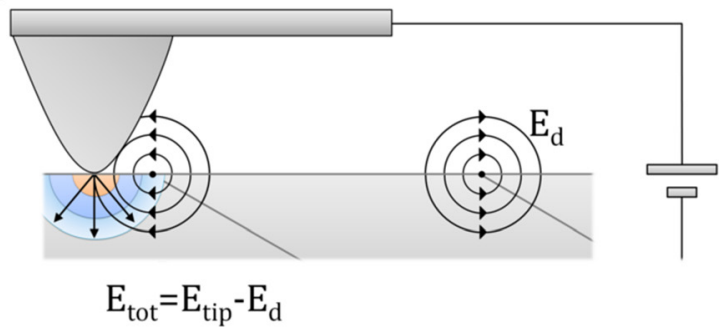

FIG. 4. Schematic illustrations of cross-sections of two domains with different out of plane polarizations (a) showing how the depolarizing field $\left(E_{d}\right)$, which occurs around the intersection of the domain wall and the free surface of the ferroelectric (marked by a black dot), can either augment the field from the cAFM tip $\left(\mathrm{E}_{\mathrm{tip}}\right)$ on one side of the domain wall (b) or diminish it on the other side (c); $\mathrm{E}_{\text {tot }}$ is the total electric field at the tip-sample contact point.

PFM data noted previously. The treatment therefore seems to have some value. Second, there is a distinct and unusual feature close to the domain wall, where the extracted polarization appears to be enhanced on one side of the wall and suppressed on the other. We do not believe that this is related to polarization variations. Rather, we suspect that it reflects local depolarizing fields, which occur at ferroelectric free surfaces adjacent to domain walls (Figure 4). These depolarizing fields are such that they act in the same sense as the applied voltage from the cAFM tip on one side of the domain wall (the side with higher out-of-plane polarization,
Figure 4(b)) and in the opposite sense on the other side (Figure 4(c)). They also increase in magnitude as the domain wall is approached. It seems, therefore, that the augmentation and diminution of field from the tip is also expressed in the intercept value of the linear $(\ln I)^{2}-V^{1 / 2}$ plots and so both polarization and local depolarizing fields might both be directly detected using cAFM. The direct measurement of local depolarizing fields is not widely described in literature, and we believe that this finding has significance.

Certainly, cAFM could be extremely useful as a complementary technique to PFM for mapping ferroelectric semiconductors. After all, cAFM information on out-of-plane polarization does not suffer the same cross-talk issues found in PFM between cantilever "flexure" (induced by in-plane polarization parallel to the cantilever axis) and genuine outof-plane tip movement. ${ }^{19}$ Moreover, our mapping suggests that $\mathrm{CAFM}$ is more a surface sensitive probe than PFM. Field distribution under the PFM tip is assumed to decay across roughly hundreds of $\mathrm{nm}$, while the tip surface contact in cAFM represents a much narrower region being probed. In Figure 5, the AFM topography illustrates the pattern of the domains at the surface, resulting from a slight preferential etching during polishing, as mentioned above. The cAFM domain maps correlate very well with the topography. However, the domain distribution that would be inferred from the PFM map of the same area is somewhat different. Presumably, the remnant of the polished grain at the sample surface is very thin and the PFM is sampling a larger signal from domain states from a different grain beneath.

In summary, by investigating polished semiconducting $\mathrm{BaTiO}_{3}$ ceramics, using scanning probe microscopy, we have demonstrated that contrast in conducting AFM is sensitive to the out-of-plane component of the ferroelectric polarization. This is consistent with the effect that polarization is expected to have on the Schottky barrier at the tip-sample interface. We have analyzed current-voltage behavior at a series of points across two adjacent stripe domains and, at each point, graphically determined a parameter, which is expected to vary with the local out-of-plane polarization. Mapping this parameter as a function of position showed not only the relative change in out-of-plane polarization that might be expected but also an apparent signature of the depolarizing fields close to the domain wall. The usefulness of transport measurements, using an AFM tip in contact with the surface, for inferring electrostatic information about the ferroelectric polarization and depolarizing fields has been therefore clearly demonstrated. (a)

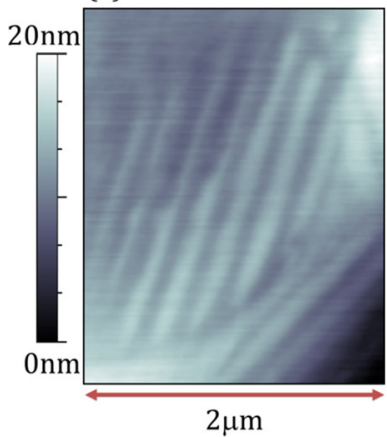

(b)

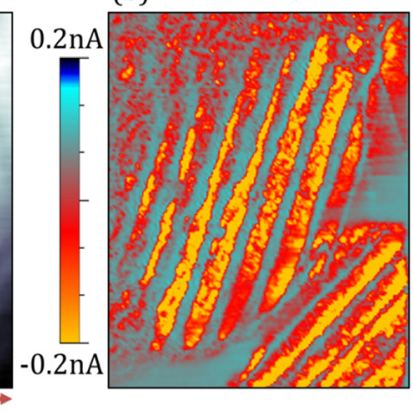

(c)

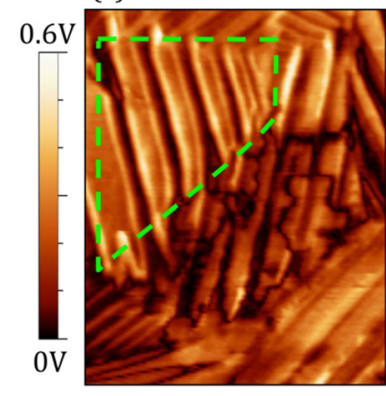

FIG. 5. In most sets of images, topography, piezoresponse, and measured current were strongly correlated. However, in some areas, the PFM domain maps ((c), displayed as amplitude only) differed from both the topography (a) and cAFM information (b) (area inside the green dashed lines in (c)). These differences are suspected to be due to the very different sampling depths associated with PFM electrostatic fields and the Schottky barriers that dictate current in $\mathrm{CAFM}$. 
The authors acknowledge Robert Twiney from Amphenol Advanced Sensors for supplying the semiconducting $\mathrm{BaTiO}_{3}$ PTCR materials, R. G. P McQuaid and M. Alexe for valuable discussions. We would also like to thank the Engineering and Physical Sciences Research Council (EPSRC) for Ph.D. studentship funding.

${ }^{1}$ G. Binnig, H. Rohrer, Ch. Gerber, and E. Weibel, Phys. Rev. Lett. 49, 57 (1982).

${ }^{2}$ G. Binnig, C. F. Quate, and Ch. Gerber, Phys. Rev. Lett. 56, 930 (1986).

${ }^{3}$ H. Béa, M. Bibes, A. Barthélémy, K. Bouzehouane, E. Jacquet, A. Khodan, J.-P. Contour, S. Fusil, F. Wyczisk, A. Forget, D. Lebeugle, D. Colson, and M. Viret, Appl. Phys. Lett. 87, 072508 (2005).

${ }^{4}$ J. Seidel, L. W. Martin, Q. He, Q. Zhan, Y.-H. Chu, A. Rother, M. E. Hawkridge, P. Maksymovych, P. Yu, M. Gajek, N. Balke, S. V. Kalinin, S. Gemming, F. Wang, G. Catalan, J. F. Scott, N. A. Spaldin, J. Orenstein, and R. Ramesh, Nat. Mater. 8, 229 (2009).

${ }^{5}$ P. Maksymovych, J. Seidel, Y.-H. Chu, P. Wu, A. P. Baddorf, L.-Q. Chen, S. V. Kalinin, and R. Ramesh, Nano Lett. 11, 1906 (2011).

${ }^{6}$ D. Meier, J. Seidel, A. Cano, K. Delaney, Y. Kumagai, M. Mostovoy, N. A. Spaldin, R. Ramesh, and M. Fiebig, Nat. Mater. 11, 284 (2012).
${ }^{7}$ J. Guyonnet, I. Gaponenko, S. Gariglio, and P. Paruch, Adv. Mater. 23, 5377 (2011)

${ }^{8}$ M. Schröder, A. Haußmann, A. Thiessen, E. Soergel, T. Woike, and L. M. Eng, Adv. Funct. Mater. 22, 3936 (2012).

${ }^{9}$ T. Sluka, A. K. Tagantsev, P. Bednyakov, and N. Setter, Nat. Commun. 4, 1808 (2013).

${ }^{10}$ M. Y. Zhuravlev, R. F. Sabirianov, S. S. Jaswal, and E. Y. Tsymbal, Phys. Rev. Lett. 94, 246802 (2005).

${ }^{11}$ V. Garcia, S. Fusil, K. Bouzehouane, S. Enouz-Vedrenne, N. D. Mathur, A. Barthélémy, and M. Bibes, Nature 460, 81 (2009).

${ }^{12}$ A. Gruverman, D. Wu, H. Lu, Y. Wang, H. W. Jang, C. M. Folkman, M. Y. Zhuravlev, D. Felker, M. Rzchowski, C. B. Eom, and E. Y. Tsymbal, Nano Lett. 9, 3539 (2009).

${ }^{13}$ A. Chanthbouala, V. Garcia, R. O. Cherifi, K. Bouzehouane, S. Fusil, X. Moya, S. Xavier, H. Yamada, C. Deranlot, N. D. Mathur, M. Bibes, A. Barthélémy, and J. Grollier, Nat. Mater. 11, 860 (2012).

${ }^{14} \mathrm{~V}$. Garcia and M. Bibes, Nat. Commun. 5, 4289 (2014).

${ }^{15}$ W. Wu, J. R. Guest, Y. Horibe, S. Park, T. Choi, S.-W. Cheong, and M. Bode, Phys. Rev. Lett. 104, 217601 (2010).

${ }^{16}$ L. Pintilie and M. Alexe, J. Appl. Phys. 98, 124103 (2005).

${ }^{17}$ L. Pintilie, I. Vrejoiu, D. Hesse, G. LeRhun, and M. Alexe, Phys. Rev. B 75, 104103 (2007).

${ }^{18}$ J. G. Simmons, Phys. Rev. Lett. 15, 967 (1965).

${ }^{19}$ S. V. Kalinin and A. Gruverman, Scanning Probe Microscopy of Functional Materials (Springer, New York, 2010), pp. 391-392. 